\title{
Host-Microbiota Co-immunity: An Intimate Relationship That Goes Beyond Protection
}

\author{
Thomas Bazin, ${ }^{* \dagger}$ Lynn Chiu, ${ }^{\ddagger}$ and Thomas Pradeu ${ }^{\ddagger \S}$
}

\begin{abstract}
Resident microorganisms, known as the microbiota, are essential for many physiological functions including protection against pathogens. Microbiota is indeed required for proper immune system development and function, and can also host-independently protect against infections. Thus, a co-constructed view of host protection involving both host and microbiota, named "co-immunity," has been proposed, and the idea of an "immunological holobiont" has been suggested. Yet this view of co-immunity might be too limited, as experimental work has shown that the immune system is involved in functions other than protection, essentially development and repair. Microbiota, through co-immunity, is thereby most likely involved in these functions, although strong evidence is currently lacking. Moreover, as our point of view is mainly host-centered, we may miss the implications of co-immunity at broader scales, including cellular and population levels. Intriguingly, coimmunity effects could be beneficial for one function and/or one level, while being detrimental for others. All these elements should be taken into consideration for microbiota manipulation, in order to avoid potentially harmful side effects.
\end{abstract}

\section{Keywords}

individuality $\bullet$ immunity $\bullet$ tissue repair $\bullet$ symbiosis $\bullet$ microbial ecology $\bullet$ colonization resistance $\bullet$ disease tolerance $\cdot$ containment

Part of a special issue, Contributed Papers from the Interdisciplinary Workshop on Holobionts, guest-edited by Derek Skillings..

*UMR 1173 INSERM/University of Versailles-Saint-Quentin, University of Paris-Saclay,

Montigny-le-Bretonneux, France, thomas.bazin@aphp.fr

$\dagger$ Department of Gastroenterology, Ambroise Paré Hospital, Assistance Publique-Hôpitaux de Paris (APHP), Boulogne Billancourt, France

$\ddagger$ UMR 1173 INSERM/University of Versailles-Saint-Quentin - University of Paris-Saclay, Montigny-le-Bretonneux, France

$\S$ Institut d'histoire and de philosophie des sciences and des techniques (UMR8590), CNRS \&

Panthéon-Sorbonne University, France

The Interdisciplinary Workshop on Holobionts was held in Bordeaux, November 6-8, 2017, and received funding from the European Research Council (ERC) under the European Union's Horizon 2020 Research and Innovation Programme, grant agreement \#637647.

Received 2 August 2018; Revised 20 February 2019; Accepted 24 February 2019 doi:10.3998/ptpbio.2097

๖ OPEN ACCESS - PTPBIO.ORG 


\section{Introduction: From Immunity against Microbes to Co-Immunity between Hosts and Microbes}

Microorganisms have long been considered as intrinsically and essentially harmful, and therefore as entities against which hosts must protect themselves. This has led to the concept that organisms isolate themselves from their microbial environment, and are protected against such microbial threats by their immune system, understood as a "defense" system (Burnet 1962; Clark 2008). Yet resident microorganisms are now clearly recognized as an integral and crucial part of our protective system against pathogens. Commensal bacteria, viruses, fungi and helminths can indeed protect their hosts against a variety of pathogens (Buffie and Pamer 2013; Belkaid and Hand 2014; Pamer 2016). We have recently proposed the label "co-immunity" for this host-microorganism partnership ensuring host protection against infectious diseases (Chiu et al. 2017).

In this short essay, we present our main arguments in favor of the idea of co-immunity, before showing that this concept must be expanded in two main directions, namely the influences of the microbiota on immune activities beyond mere protection, and on multiple levels of immune responses.

A few remarks about the vocabulary used in the present paper are in order. We define "microbiota" as all the resident microorganisms living in or on a host, regardless of the nature of their ecological interaction (parasitic, commensal, and mutualistic) with the host, their size, or taxonomic category (bacteria, viruses, fungi, or parasites such as helminths). In addition, we understand the notion of "holobiont" physiologically, as the collective entity composed by the host and its resident microbiota and displaying functions (in a systemic sense, Cummins 1975) realized jointly by the host and the microbiota (Chiu and Eberl 2016; Pradeu 2016). We do not claim that this collective entity is an evolutionary unit, contrary to the dominant (and disputed) use of the term "holobiont" (Vandenkoornhuyse et al. 2015; Bordenstein and Theis 2015; Douglas and Werren 2016; Skillings 2016; Doolittle and Booth 2017; Roughgarden et al. 2017), though it could qualify as an ecological unit (Hester et al. 2016). Moreover, the crux of our arguments concerns the details of how the microbiota influences immunity and these arguments apply to the intimate association between a host and its microbiota, whether or not one considers that such association qualifies as a "holobiont."

\section{What Is "Co-immunity"?}

In a recent paper (Chiu et al. 2017), we have proposed the concept of "co-immunity." This concept builds on previous knowledge about the potentially protective role of the microbiota (dealing mainly with localized colonization resistance), but also makes several steps further. Recent work has distinguished two main modes of microbiota-induced protection: a direct one via microbe-to-microbe interactions and an indirect one via activation by the microbiota of a host's immune system. For instance, probiotic Escherichia coli (Nissle 1917) directly outcompetes commensal and pathogen Escherichia coli and Salmonella typhimurium via micromicin secretion (Sassone-Corsi et al. 2016), while protozoan Tritrichomonas musculis indirectly protects from gut $S$. typhimurium infection through the promotion of inflammatory Th1 and Th17 type immunity (Chudnovskiy et al. 2016). These two modes of protection are grouped together under the concept of "colonization resistance," which refers to all forms of host-dependent or hostindependent resistance to pathogens that are induced by the microbiota. 
Still, this classic concept of microbiota-induced protection is too narrow for two reasons: on the one hand, the concept of colonization resistance is generally limited to a single protection strategy - that is, the resistance against pathogens' establishment and growth; on the other, protective effects are limited to the immediate vicinity of protective microbes. Thus, colonization resistance seems too limited to embrace the variety and complexity of microbiota-mediated protective effects.

First, other protective mechanisms than colonization resistance exist, namely containment and disease tolerance. Containment is the controlled localization of microbes within a particular site inside the host. For instance, in various organisms, bacteriophages that adhere to mucus glycoproteins can prevent translocation of bacteria, thus providing a host-independent localized protection of mucosal surfaces against bacterial infections (Barr et al. 2013). Disease tolerance is the limitation of host's tissue damage induced by pathogens, without pathogen elimination. As an illustration, Bacteroides fragilis in the gut affects systemic T-cell responses through the action of the bacterium-derived polysaccharide A, which protects against pathobiont Helicobater hepaticus colitis via the production of anti-inflammatory IL-10 by $\mathrm{CD}_{4}{ }^{+} \mathrm{T}$ cells and the promotion of regulatory $\mathrm{T}$ cells, without any effect on pathogen fitness or load (Mazmanian, Round, and Kasper 2008).

Second, microorganisms in one organ (mainly the gut) can influence immune responses in other organs. Long-reaching protective effects are less documented than local effects in the scientific literature, but convincing examples exist. For instance, mice challenged with pulmonary staphylococcal infection but lacking segmented filamentous bacteria in their gut microbiota show more severe infection with higher bacterial load and mortality, associated with diminished lung concentration of Th17 immune effector cells. Reintroduction of segmented filamentous bacteria is sufficient to restore protective effect (Gauguet et al. 2015).

Thus, we propose a systematic classification of protective mechanisms that includes a broader range of protective strategies than localized colonization resistance, distinguishing the modes (direct or indirect), the ranges (localized or long-reaching) and the effects (mechanisms) of protection (see Figure 1). The notion of co-immunity that we propose (i.e., a form of immune protection associating components of several organisms), which in the present contexts combines components of the host and of the microbiota, includes all these different modes, ranges, and effects.

\section{Enriching the Concept of Co-Immunity in Terms of Activities and Levels}

Despite the encompassing nature of the concept of co-immunity, we think it is still too limited, and must therefore be expanded in two main directions. First, immunity is not limited to protective strategies, and taking this fact into account extends significantly the implications of the concept of co-immunity. Second, co-immunity might concern other levels than just the individual organism.

\section{Co-immunity beyond protection}

We previously defined co-immunity as a form of immune protection (Chiu et al. 2017). But what does "defense" exactly mean in this context? If we agree that to defend means to fight or to help to fight against an external aggression, which is the commonly accepted definition, colonization resistance and containment can be considered as defense mechanisms, acting as "knights"- either using a "shield" (defense) or a "sword" (attack). But should disease tolerance be always considered as a defense mechanism? In the case of the inhibition of pathogenic tox- 


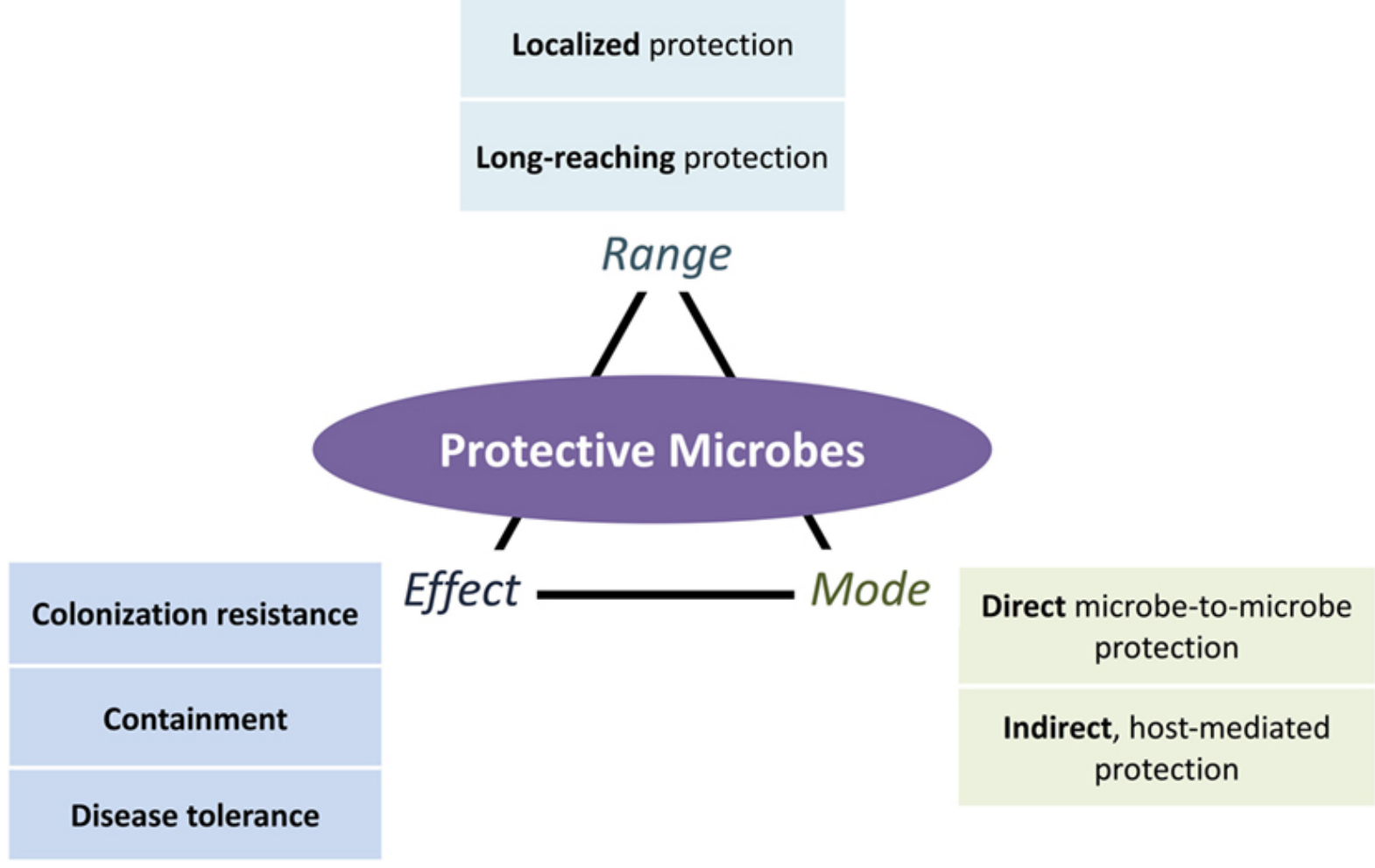

Figure 1: An expanded view of microbe-conferred protection. Microorganisms can protect the host in different ways, depending on the mode of protection (direct ecological and indirect host-mediated), the effects of protection (colonization resistance against pathogenic establishment and growth, containment of pathogens and their effects, and disease tolerance of pathogens while suppressing their negative effects), and the range of protection (localized or long-reaching, with the latter further divided into protection that is systemic or from one locale to another locale). All three aspects of protection can occur in combination. Long-reaching protection, for instance, involves both direct (ecological) and indirect (host-mediated) modes of protection that result in colonization resistance, containment, or disease tolerance effects. Source: Frontiers in Immunology (Chiu et al. 2017).

ins, for instance (de Sablet et al. 2009), it might be said so. But the toleration (rather than the destruction) of pathogens can be a cost-effective alternative when upkeep of pathogen fitness does not strongly decrease host fitness (e.g., when pathogen virulence is low), especially since the side effects of aggressive inflammatory immune responses are sometimes more damaging to the host than the "infection" itself. For instance, gut commensal Escherichia coli O21:H+ antagonizes muscle wasting during Burkholderia thailandensis or Salmonella typhimurium infections, without a negative impact on the fitness of these pathogens (Schieber et al. 2015). In this precise case, disease tolerance could act more like a wise gate-keeper than like a knight, away from a strictly speaking defense action, and sometimes paving the way for a potentially mutualistic relationship. Thus, it is more accurate to consider co-immunity as a form of immune protection against pathogens.

Moreover, we know that immunity is implicated in many other activities than protection alone. The immune system plays indeed a key role in development and tissue repair (Laurent et al. 2017; Truchetet and Pradeu 2018). Could co-immunity be implicated in developmentassociated and repair-associated activities?

The notion that animal development universally relies on microbial cues (developmental symbiosis) is gaining traction (Gilbert 2016). However, in most cases the three actors' game 
between microbiota, immune system and development remains to be demonstrated, as for the most part what we have is correlations only. A much-studied bacterial symbiosis in the animal world is the association between the squid Euprymna scolopes and the bacterium Vibrio fischeri, where a crucial step of the squid's organogenesis relies on the presence of this particular bacterium. $V$. fischeri is specifically required for the E. scolopes's light organ to fully develop, which is to lose its superficial ciliated field. This action is linked with a direct interaction between tracheal cytotoxin (a fragment of the peptidoglycan) released by $V$. fischeri and epithelium-lined crypts of the light organ (Koropatnick et al. 2004). Pathways explaining developmental effects distant from this specific epithelium probably involve macrophage-like hemocytes that are recruited during $V$. fischeri colonization (McFall-Ngai, Nyholm, and Castillo 2010), suggesting that in the present case organogenesis relies on the interaction between a specific bacterium and the animal's immune system. Interestingly, microscopic examination of various squid tissues revealed that host hemocytes specifically migrate into the epithelial fields on the surface of the light organ before any signal of morphogenesis changes, suggesting a causal link between hemocyte modifications and organogenesis. Moreover, hemocyte gene expression correlates with the induction of light organ morphogenesis, suggesting that bacteria-induced molecular changes in the hemocytes are required for their developmental effects. However, direct impact of $V$. fischeri on hemocytes' gene expression has yet to be demonstrated (Koropatnick, Kimbell, and McFall-Ngai 2007).

Microbiota in association with the immune system is also suspected to play a role in the development and the organization of the enteric nervous system (Obata and Pachnis 2016). For instance, in the network of the murine enteric nervous system, mucosal glial cells develop in response to gut microbiota, but it is unclear whether this is a consequence of direct glia sensing of microbial factors, or if it is instead mediated by the immune system (Veiga-Fernandes and Pachnis 2017). In humans, the microbiota is suspected to play a role in neurodevelopment, this having been particularly studied in autism (Kelly et al. 2017). Microglia development, maturation and function are influenced both prenatally and postnatally by the microbiota (Erny et al. 2015); as microglia contribute to brain development, microbiota could indirectly influence brain development (Thion et al. 2018), though more research is needed to entirely establish this claim.

Concerning repair, some studies show a beneficial role of particular species in healing intestinal ulcers and cutaneous infections (Lukic et al. 2017), potentially through systemic effects of beneficial bacteria, whereas others point to an accelerated and scarless skin healing in germ-free conditions (Canesso et al. 2014). Interestingly, the three-actors' game between the microbiota, the immune system, and repair processes has begun to be deciphered. A link between the microbiota and intestinal repair was suspected after the discovery that toll-like receptor (TLR) activation by microbiota components is needed to promote tissue repair and host survival following acute injury (Rakoff-Nahoum et al. 2004). Moreover, Lai et al. showed that lipoteichoic acid, produced by skin commensal Staphylococcus epidermidis, can bind to TLR2, and thus mitigate inflammation, thereby promoting wound healing (Lai et al. 2009). More recently, Linehan et al. showed that a skin commensal can induce a specific $\mathrm{T}$-cell response that accelerates skin wound healing, proving the implication of the immune system in microbiota modulation of tissue repair (Linehan et al. 2018). Notably, commensal-specific T-cells expressed repair signature genes and were different from pathogen-induced ones.

Together, these results suggest that the boundaries of our conceptual framework need to be extended, and raise a new question: If a microbe can modulate different immune functions, is there a possibility that what we call a pathogen because of its deleterious effect on one function could be beneficial in another immune function? And, conversely, can a protective microbe for 
one function be deleterious for another function? The latter is crucial for the safety of microbiota interventions.

\section{Co-immunity beyond the level of the organism}

Our vision of protection is usually centered on the level of the organism. Yet co-immunity could occur at different scales, both within and beyond the organism. This raises the question of whether pathogenic effects can impact differentially the various levels under consideration.

At the population scale, microbiota transmission could be implicated in social immunity in insects (Cremer, Armitage, and Schmid-Hempel 2007), and in "herd immunity" (Fine 1993) in humans and cattle, whose prototypical example is vaccination. Social immunization-that is, the inter-individual transmission of protective properties against pathogens-is a part of social immunity and has been observed in Zootermopsis angusticollis termite (Traniello, Rosengaus, and Savoie 2002) and in Bombus terrestris bumblebee (Sadd et al. 2005). The precise mechanisms are unknown, and they cannot involve antibody transmission in invertebrate animals (Moret and Schmid-Hempel 2001). As microbiota transmission is strongly favored by sociality (Kwong and Moran 2016), it could be the vector of this sort of social immunity.

In humans, the carcinogenic effect of Helicobacter pylori on gastric mucosa is well-known, but at the organism scale $H$. pylori infection is associated with a protection against tuberculosis (Perry et al. 2010), which can hypothetically modify the transmission of infection at the population level. It is also plausible that a peculiar component of the microbiota could protect one host from the damages induced by a pathogen, for instance by inducing disease tolerance, thereby encouraging its growth and transmission and decreasing population's fitness, though evidence for such a phenomenon is currently lacking. In the opposite way, a particular component of the microbiota could increase community fitness, while disadvantaging predisposed hosts (altruistic immunity).

Within a multicellular organism, it is perfectly plausible that a bacterium hosts a phage that protects the bacterium against pathogens (which means that there is a co-immunity between the bacterium and the phage), but this association weakens the immune protection of the multicellular host against a given pathogenic threat in a given context. It is also conceivable (though no evidence currently exists for this, to our knowledge) that an intracellular microorganism increases cell-intrinsic immunity (i.e., immunity at the level of a cell, e.g., Bieniasz 2004; Yan and Chen 2012; Ablasser et al. 2013), at the expense of the immune response of the whole organism against a given pathogen. This could happen in plants, for example, where immunity is to a large extent dependent on cell-intrinsic processes, making the coordination of these cellular responses at the higher level of the organism quite complicated (Szittya and Burgyán 2013). Other interesting cases of conflicts between levels of co-immunity could include organisms that transition from a unicellular to a multicellular stage, with the emergence of collective immunity at the multicellular level, like Dictyostelium discoideum (Chen, Zhuchenko, and Kuspa 2007). Here again, one could imagine an intracellular symbiont favoring the immune protection of a cell within the multicellular slug, but detrimental to immune protection of the slug as a whole against a given pathogen. Though speculative, these considerations are plausible, and it is likely that the lack of evidence about such phenomena is to a large extent due to lack of investigations based on these exact questions. 


\section{Conclusion}

The immune system is so dependent on its interactions with the microbiota for its development and functioning that the "pure" eukaryote-only concept of immunity seems to progressively vanish. In parallel, "host-independent" immune protection is very relative, as microbiota composition is actively modulated by the immune system. Thus, it seems essential to adopt a co-constructed view of immunity (according to which immunity is the product of the intricate contribution of both the host and the microbiota).

An open and perhaps slightly provocative question is whether it is useful to continue to use the term "immunity" alone, or if this term should be completely replaced by that of "coimmunity" (see Figure 2). It becomes increasingly clear that the microbiota has some influence

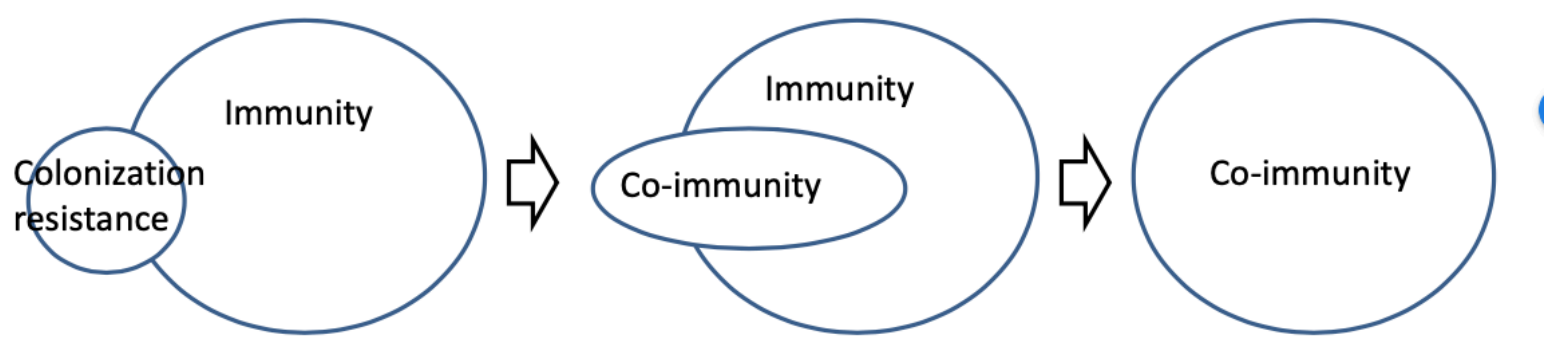

Figure 2: Conceptual transition from colonization resistance as a peripheral part of our understanding of immunity to co-immunity as an integrated sub-part of immunity, and finally to co-immunity alone.

on all types of immunological responses (including different cell types, different bodily compartments, and different immunological "functions"-including protection, repair, and development). Our expanded view of co-immunity gives credence to the notion of an "immunological holobiont" (Pradeu 2016; Postler and Ghosh 2017), without again assuming that this entity constitutes an evolutionary unit.

It might be possible to object to our argument here that the vision of the immune system as multifunctional is not consensual, and that evidence concerning development and repair mediated by a dialogue between the immune system and the microbiota is scarce. In response, we would say that recent research does seem to point in that direction and that, as philosophers and medical doctors involved in microbiota research, our aim is mainly to try to open up original avenues for future research. We suggest that testing the potential beneficial effects of a new bug should be accompanied by a broad reflection about its impact on all immune functions (not just protection against infections), at different scales (not just that of the organism), and in different contexts. This expanded view of co-immunity should also be combined with a switch from a host-centric view (as is usually the case in discussions over "holobionts") to a microbe-centric view. This switch is likely to further enrich the co-constructed view of immunity that we have tried to propose in this essay.

\section{Acknowledgments}

We would like to thank Nadine Cerf-Bensussan, Laurence Delhaes, Gérard Eberl, Bruno Lemaitre, Thierry Schaeverbeke, Philippe Sansonetti, and Marie-Elise Truchetet for discussions. L. C. and T. P. 
have received funding from the European Research Council (ERC) under the European Union's Horizon 2020 research and innovation programm, grant agreement $n^{\circ} 637647$ - IDEM.

\section{Literature cited}

Ablasser, Andrea, Jonathan L. Schmid-Burgk, Inga Hemmerling, Gabor L. Horvath, Tobias Schmidt, Eicke Latz, and Veit Hornung. 2013. "Cell Intrinsic Immunity Spreads to Bystander Cells via the Intercellular Transfer of cGAMP.” Nature 503 (7477): 530-534. https://doi.org/10.1038/ nature12640.

Barr, Jeremy J., Rita Auro, Mike Furlan, Katrine L. Whiteson, Marcella L. Erb, Joe Pogliano, Aleksandr Stotland, et al. 2013. "Bacteriophage Adhering to Mucus Provide a Non-Host-Derived Immunity.” Proc. Natl. Acad. Sci. U.S.A. 110 (26): 10771-10776. https://doi.org/10.1073/pnas. 1305923110.

Belkaid, Yasmine, and Timothy W. Hand. 2014. "Role of the Microbiota in Immunity and Inflammation.” Cell 157 (1): 121-141. https://doi.org/10.1016/j.cell.2014.03.011.

Bieniasz, Paul D. 2004. “Intrinsic Immunity: A Front-Line Defense Against Viral Attack.” Nat Immunol 5 (11): 1109-1115. https://doi.org/10.1038/ni1125.

Bordenstein, Seth R., and Kevin R. Theis. 2015. "Host Biology in Light of the Microbiome: Ten Principles of Holobionts and Hologenomes.” PLoS Biol 13 (8): e1002226. https://doi.org/10.1371/ journal.pbio.1002226.

Buffie, Charlie G., and Eric G. Pamer. 2013. "Microbiota-Mediated Colonization Resistance Against Intestinal Pathogens.” Nat. Rev. Immunol. 13 (11): 790-801. https://doi.org/10.1038/nri3535.

Burnet, Frank M. 1962. The Integrity of the Body: A Discussion of Modern Immunological Ideas. Cambridge, MA: Harvard University Press.

Canesso, Maria C. C., Angélica T. Vieira, Tiago B. R. Castro, Brígida G. A. Schirmer, Daniel Cisalpino, Flaviano S. Martins, Milene A. Rachid, et al. 2014. "Skin Wound Healing Is Accelerated and Scarless in the Absence of Commensal Microbiota." J. Immunol. 193 (10): 5171-5180. https://doi.org/10.4049/jimmunol.1400625.

Chen, Guokai, Olga Zhuchenko, and Adam Kuspa. 2007. "Immune-Like Phagocyte Activity in the Social Amoeba.” Science 317 (5838): 678-681. https://doi.org/10.1126/science.1143991.

Chiu, Lynn, Thomas Bazin, Marie-Elise Truchetet, Thierry Schaeverbeke, Laurence Delhaes, and Thomas Pradeu. 2017. "Protective Microbiota: From Localized to Long-Reaching CoImmunity.” Front. Immunol. 8. https://doi.org/10.3389/fimmu.2017.01678.

Chiu, Lynn, and Gérard Eberl. 2016. "Microorganisms as Scaffolds of Host Individuality: An EcoImmunity Account of the Holobiont." Biol Philos 31 (6): 819-837. https://doi.org/10.1007/ s10539-016-9552-0.

Chudnovskiy, Aleksey, Arthur Mortha, Veronika Kana, Andrea Kennard, Juan David Ramirez, Adeeb Rahman, Romain Remark, et al. 2016. "Host-Protozoan Interactions Protect From Mucosal Infections Through Activation of the Inflammasome." Cell 167 (2): 444-456.e14. https://doi.org/ 10.1016/j.cell.2016.08.076.

Clark, William R. 2008. In Defense of Self: How the Immune System Really Works. New York: Oxford University Press.

Cremer, Sylvia, Sophie A. O. Armitage, and Paul Schmid-Hempel. 2007. "Social Immunity." Curr. Biol. 17 (16): R693-702. https://doi.org/10.1016/j.cub.2007.06.008. 
Cummins, Robert. 1975. "Functional Analysis.” Journal of Philosophy 72 (20): 741-765. https://doi.org/ $10.2307 / 2024640$.

De Sablet, Thibaut, Christophe Chassard, Annick Bernalier-Donadille, Marjolaine Vareille, Alain P. Gobert, and Christine Martin. 2009. "Human Microbiota-Secreted Factors Inhibit Shiga Toxin Synthesis by Enterohemorrhagic Escherichia coli O157: H7.” Infect. Immun. 77 (2): 783-790. http s://doi.org/10.1128/1A1.01048-08.

Doolittle, W. Ford, and Austin Booth. 2017. "It's the Song, Not the Singer: An Exploration of Holobiosis and Evolutionary Theory." Biol Philos 32 (1): 5-24. https://doi.org/10.1007/s10539-0169542-2.

Douglas, Angela E., and John H. Werren. 2016. "Holes in the Hologenome: Why Host-Microbe Symbioses Are Not Holobionts.” mBio 7 (2): e02099-15. https://doi.org/10.1128/mBio.0209915.

Erny, Daniel, Anna Lena Hraběde Angelis, Diego Jaitin, Peter Wieghofer, Ori Staszewski, Eyal David, Hadas Keren-Shaul, et al. 2015. "Host Microbiota Constantly Control Maturation and Function of Microglia in the CNS.” Nat. Neurosci. 18 (7): 965-977. https://doi.org/10.1038/nn. 4030 .

Fine, Paul E. M. 1993. “Herd Immunity: History, Theory, Practice.” Epidemiol Rev 15 (2): 265-302. https://doi.org/10.1093/oxfordjournals.epirev.a036121.

Gauguet, Stefanie, Samantha D'Ortona, Kathryn Ahnger-Pier, Biyan Duan, Neeraj K. Surana, Roger $\mathrm{Lu}$, Colette Cywes-Bentley, et al. 2015. "Intestinal Microbiota of Mice Influences Resistance to Staphylococcus Aureus Pneumonia.” Infect. Immun. 83 (10): 4003-4014. https://doi.org/10.1128/ IAI.00037-15.

Gilbert, Scott F. 2016. "Developmental Plasticity and Developmental Symbiosis: The Return of EcoDevo.” Curr. Top. Dev. Biol. 116:415-433. https://doi.org/10.1016/bs.ctdb.2015.12.006.

Hester, Eric R., Katie L. Barott, Jim Nulton, Mark Ja Vermeij, and Forest L. Rohwer. 2016. "Stable and Sporadic Symbiotic Communities of Coral and Algal Holobionts.” ISME J 10 (5): 11571169. https://doi.org/10.1038/ismej.2015.190.

Kelly, John R., Chiara Minuto, John F. Cryan, Gerard Clarke, and Timothy G. Dinan. 2017. "Cross Talk: The Microbiota and Neurodevelopmental Disorders." Front Neurosci 11:490. https://doi. org/10.3389/fnins.2017.00490.

Koropatnick, Tanya A., Jacquelyn T. Engle, Michael A. Apicella, Eric V. Stabb, William E. Goldman, and Margaret J. Mcfall-Ngai. 2004. "Microbial Factor-Mediated Development in a HostBacterial Mutualism.” Science 306 (5699): 1186-1188. https://doi.org/10.1126/science.1102218.

Koropatnick, Tanya A., Jennifer R. Kimbell, and Margaret J. Mcfall-Ngai. 2007. "Responses of Host Hemocytes During the Initiation of the Squid-Vibrio Symbiosis.” Biol. Bull. 212 (1): 29-39. http s://doi.org/10.2307/25066578.

Kwong, Waldan K., and Nancy A. Moran. 2016. "Gut Microbial Communities of Social Bees." Nat. Rev. Microbiol. 14 (6): 374-384. https://doi.org/10.1038/nrmicro.2016.43.

Lai, Yuping, Anna Di Nardo, Teruaki Nakatsuji, Anke Leichtle, Yan Yang, Anna L. Cogen, Zi-Rong Wu, et al. 2009. "Commensal Bacteria Regulate Toll-Like Receptor 3-Dependent Inflammation After Skin Injury.” Nat. Med. 15 (12): 1377-1382. https://doi.org/10.1038/nm.2062.

Laurent, Paôline, Valérie Jolivel, Pauline Manicki, Lynn Chiu, Cécile Contin-Bordes, Marie-Elise Truchetet, and Thomas Pradeu. 2017. "Immune-Mediated Repair: A Matter of Plasticity." Front. Immunol. 8. https://doi.org/10.3389/fimmu.2017.00454. 
Linehan, Jonathan L., Oliver J. Harrison, Seong-Ji Han, Allyson L. Byrd, Ivan Vujkovic-Cvijin, Alejandro V. Villarino, Shurjo K. Sen, et al. 2018. "Non-Classical Immunity Controls Microbiota Impact on Skin Immunity and Tissue Repair." Cell 172 (4): 784-796.e18. https://doi.org/10. 1016/j.cell.2017.12.033.

Lukic, Jovanka, Vivien Chen, Ivana Strahinic, Jelena Begovic, Hadar Lev-Tov, Stephen C. Davis, Marjana Tomic-Canic, et al. 2018. "Probiotics or Pro-Healers the Role of Beneficial Bacteria in Tissue Repair.” Wound Repair Regen, https://doi.org/10.1111/wrr.12607.

Mazmanian, Sarkis K., June L. Round, and Dennis L. Kasper. 2008. “A Microbial Symbiosis Factor Prevents Intestinal Inflammatory Disease.” Nature 453 (7195): 620-625. https://doi.org/10.1038/ nature07008.

Mcfall-Ngai, Margaret, Spencer V. Nyholm, and Maria G. Castillo. 2010. "The Role of the Immune System in the Initiation and Persistence of the Euprymna Scolopes-Vibrio Fischeri Symbiosis." Semin. Immunol. 22 (1): 48-53. https://doi.org/10.1016/j.smim.2009.11.003.

Moret, Yannick, and Paul Schmid-Hempel. 2001. "Entomology: Immune Defence in Bumble-Bee Offspring.” Nature 414 (6863): 506. https://doi.org/10.1038/35107138.

Obata, Yuuki, and Vassilis Pachnis. 2016. "The Effect of Microbiota and the Immune System on the Development and Organization of the Enteric Nervous System.” Gastroenterology 151 (5): 836844. https://doi.org/10.1053/j.gastro.2016.07.044.

Pamer, Eric G. 2016. "Resurrecting the Intestinal Microbiota to Combat Antibiotic-Resistant Pathogens.” Science 352 (6285): 535-538. https://doi.org/10.1126/science.aad9382.

Perry, Sharon, Bouke C. De Jong, Jay V. Solnick, Maria De La Luz Sanchez, Shufang Yang, Philana Ling Lin, Lori M. Hansen, et al. 2010. "Infection With Helicobacter pylori Is Associated With Protection Against Tuberculosis.” PLoS ONE 5 (1): e8804. https://doi.org/10.1371/journal.pone. 0008804.

Postler, Thomas Siegmund, and Sankar Ghosh. 2017. "Understanding the Holobiont: How Microbial Metabolites Affect Human Health and Shape the Immune System." Cell Metab 26 (1): 110-130. https://doi.org/10.1016/j.cmet.2017.05.008.

Pradeu, Thomas. 2016. "Organisms or Biological Individuals? Combining Physiological and Evolutionary Individuality.” Biol Philos 31 (6): 797-817. https://doi.org/10.1007/s10539-016-9551-1.

Rakoff-Nahoum, Seth, Justin Paglino, Fatima Eslami-Varzaneh, Stephen Edberg, and Ruslan Medzhitov. 2004. "Recognition of Commensal Microflora by Toll-Like Receptors Is Required for Intestinal Homeostasis.” Cell 118 (2): 229-241. https://doi.org/10.1016/j.cell.2004.07.002.

Roughgarden, Joan, Scott F. Gilbert, Eugene Rosenberg, Ilana Zilber-Rosenberg, and Elisabeth A. Lloyd. 2017. "Holobionts as Units of Selection and a Model of Their Population Dynamics and Evolution.” Biol Theory, 1-22. https://doi.org/10.1007/s13752-017-0287-1.

Sadd, Ben M., Yvonne Kleinlogel, Regula Schmid-Hempel, and Paul Schmid-Hempel. 2005. "TransGenerational Immune Priming in a Social Insect.” Biol Lett 1 (4): 386-388. https://doi.org/10. 1098/rsbl.2005.0369.

Sassone-Corsi, Martina, Sean-Paul Nuccio, Henry Liu, Dulcemaria Hernandez, Christine T. Vu, Amy A. Takahashi, Robert A. Edwards, et al. 2016. "Microcins Mediate Competition Among Enterobacteriaceae in the Inflamed Gut.” Nature 540 (7632): 280-283. https://doi.org/10.1038/ nature20557. 
Schieber, Alexandria M. Palaferri, Yujung Michelle Lee, Max W. Chang, Mathias Leblanc, Brett Collins, Michael Downes, Ronald M. Evans, et al. 2015. "Disease Tolerance Mediated by Microbiome E. coli Involves Inflammasome and IGF-1 Signaling.” Science 350 (6260): 558-563. https://doi.org/10.1126/science.aac6468.

Skillings, Derek. 2016. "Holobionts and the Ecology of Organisms: Multi-Species Communities or Integrated Individuals?” Biol Philos 31 (6): 875-892. https://doi.org/10.1007/s10539-016-95440 .

Szittya, György, and József Burgyán. 2013. "RNA Interference-Mediated Intrinsic Antiviral Immunity in Plants." Curr. Top. Microbiol. Immunol. 371:153-181. https://doi.org/10.1007/978-3-64237765-5_6.

Thion, Morgane Sonia, Donovan Low, Aymeric Silvin, Jinmiao Chen, Pauline Grisel, Jonas SchulteSchrepping, Ronnie Blecher, et al. 2018. "Microbiome Influences Prenatal and Adult Microglia in a Sex-Specific Manner.” Cell 172 (3): 500-516.e16. https://doi.org/10.1016/j.cell.2017.11.042.

Traniello, James F. A., Rebeca B. Rosengaus, and Keely Savoie. 2002. "The Development of Immunity in a Social Insect: Evidence for the Group Facilitation of Disease Resistance." Proc Natl Acad Sci USA 99 (10): 6838-6842. https://doi.org/10.1073/pnas.102176599.

Truchetet, Marie-Elise, and Thomas Pradeu. 2018. "Redundancy and Robustness in Immunity." Seminars in Immunology 36:45-55. https://doi.org/10.1016/j.smim.2018.02.013.

Vandenkoornhuyse, Philippe, Achim Quaiser, Marie Duhamel, Amandine Le Van, and Alexis Dufresne. 2015. "The Importance of the Microbiome of the Plant Holobiont." New Phytol 206 (4): 1196-1206. https://doi.org/10.1111/nph.13312.

Veiga-Fernandes, Henrique, and Vassilis Pachnis. 2017. "Neuroimmune Regulation During Intestinal Development and Homeostasis.” Nat. Immunol. 18 (2): 116-122. https://doi.org/10.1038/ni. 3634 .

Yan, Nan, and Zhijian J. Chen. 2012. "Intrinsic Antiviral Immunity." Nat Immunol 13 (3): 214-222. https://doi.org/10.1038/ni.2229.

(C) 2022 Author(s)

This is an open-access article distributed under the terms of the Creative Commons Attribution 4.0 International license, which permits anyone to download, copy, distribute, display, or adapt the text without asking for permission, provided that the creator(s) are given full credit.

ISSN 2475-3025 